\title{
Investigating the Origin of Luminescence in Zinc Oxide Nanostructures With STEM-Cathodoluminescence
}

\author{
Edward R. White ${ }^{1}$, Ashley Howkins ${ }^{2}$, Charlotte K. Williams ${ }^{1}$, and Milo S. P. Shaffer ${ }^{1}$ \\ 1. Department of Chemistry, Imperial College London, South Kensington, London, SW7 2AZ, UK. \\ 2. Experimental Techniques Centre, Brunel University London, Uxbridge, UB8 3PH, UK.
}

$\mathrm{ZnO}$ nanostructures display luminescence in the UV and across the visible spectrum, and show promise as future nanoscale electronic, optoelectronic, and sensing devices [1]. The visible luminescence arises from surface or bulk states at energies inside the $\mathrm{ZnO}$ bandgap, however, a fundamental understanding of the luminescent sources is still lacking. The assignment of particular defects to different visible emission peaks is a highly controversial and active area of current research [1,2]. Here, we perform the first spatially resolved scanning transmission electron microscopy cathodoluminescence (STEM-CL) measurements on $\mathrm{ZnO}$ nanostructures, and show the emergence of CL spectral peaks associated with morphological changes in $\mathrm{ZnO}$ nanorods. Further studies using parallel techniques in the TEM sensitive to intrinsic and extrinsic defects (e.g. HRTEM, atomic resolution HAADF-STEM, EELS, and EDS) will likely conclusively reveal the origin of emission in $\mathrm{ZnO}$ and other technologically relevant, luminescent nanostructures.

Unlike photoluminescence, cathodoluminescence can probe the emission properties of individual nanostructures since the electron beam can be condensed to nanometer scale. SEM-CL has been used to study defect emission in individual $\mathrm{ZnO}$ structures previously, with studies showing, for example, green emission associated with $\mathrm{Zn}$ vacancies [2] and a blueshift in the near-band-edge emission with decreasing particle size[3]. However, SEM cannot resolve atomic features to correlate with emission changes, as SEM resolution is limited to a few nanometers. Cathodoluminescence measurements done in the TEM have this unique capability.

Here, we use a Gatan Vulcan STEM-CL system to measure changes in cathodoluminescence spectra within individual $\mathrm{ZnO}$ nanostructures. Figure 1 shows STEM images and a CL spectrum image acquired across an $800 \mathrm{~nm}$ long $\mathrm{ZnO}$ nanorod. The spectrum image contains 100 individual spectra, each acquired as the electron beam rasters over an area $8 \mathrm{~nm}$ long $\times$ the width of the nanorod $(50 \mathrm{~nm})$. The emission onset at $375 \mathrm{~nm}$ is consistent with the near-band-edge in $\mathrm{ZnO}$ [1]. Comparison of the CL spectrum image and the STEM images of the same rod illustrates the dramatic changes in visible emission with morphological changes in the nanorod.

The most obvious spectral feature in Figure $1 \mathrm{~d}$ is the orange emission at $\sim 600 \mathrm{~nm}$. Previously, orange emission has been attributed to surface dislocations, oxygen interstitials, and zinc vacancies; a definitive source remains elusive [1]. Comparing Figures 1c-d it is evident the orange emission is coincident with an increase in HAADF signal. Figure 2 illustrates this more clearly; the amplitude of Gaussians fit to the UV near-band-edge and orange deep-level emissions are plotted as a function of distance along the rod. Other CL spectral peaks also correlate with structural features in the nanorod. There is strong blue emission centered at $\sim 450 \mathrm{~nm}$ associated with the tip of the nanorod. There is weak, broad emission centered at $\sim 435 \mathrm{~nm}$ in the region with the strong emission at $600 \mathrm{~nm}$. Finally there is weak, but sharp, violet emission at $400 \mathrm{~nm}$, associated with strain in lower portion of the rod, visible in the ADF STEM 
image (Figure 1b). Correlating these spectral peaks and morphological changes with further defect investigations promises to reveal the origin of visible luminescence in $\mathrm{ZnO}$ nanostructures [4].

\section{References:}

[1] AB Djurisić and YH Leung, Small 2 (2006), p. 944.

[2] F Fabbri et al, Scientific Reports 4 (2014), p. 5158.

[3] CW Chen et al, Applied Physics Letters 88 (2006), p. 241905.

[4] The authors acknowledge funding from EPSRC grant number EP/K035274/1, and Brunel University and the Experimental Techniques Centre for funding and making available the STEM-CL system, respectively.

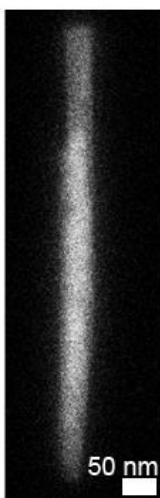

(a)

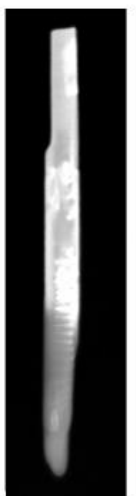

(b)

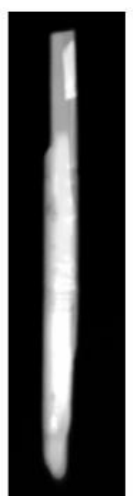

(c)

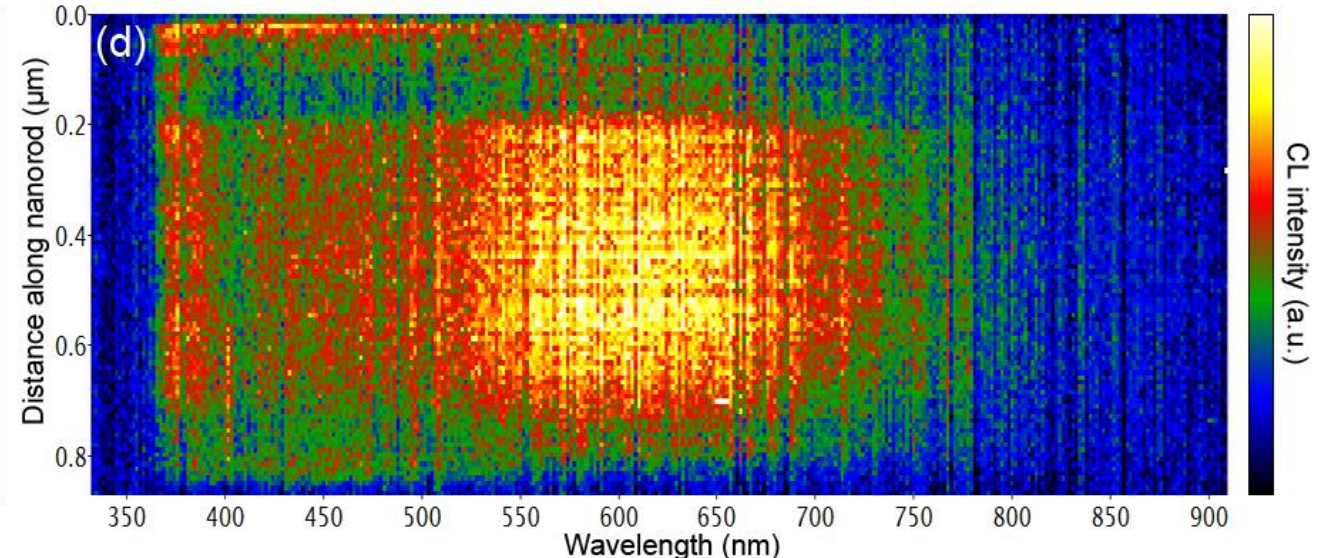

Figure 1. STEM images and CL spectrum image acquired along the length of a $\mathrm{ZnO}$ nanorod. Simultaneously acquired (a) panchromatic CL, (b) ADF, and (c) HAADF images. (d) CL spectrum image of the $\mathrm{ZnO}$ nanorod pictured in (a)-(c).
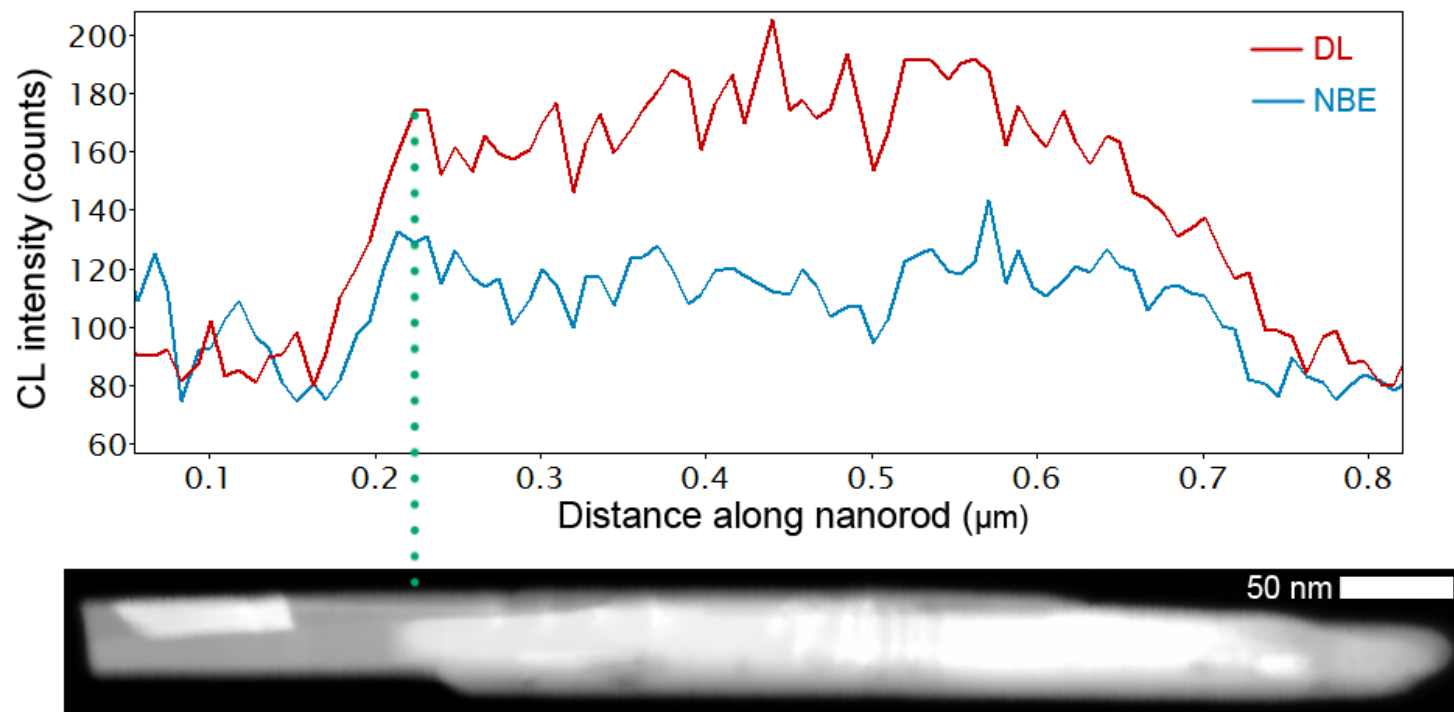

Figure 2. Increase in deep-level (DL) emission intensity relative to the near-band-edge (NBE) intensity associated with morphological changes in the $\mathrm{ZnO}$ nanorod. (top) Plot of emission intensity vs. distance along the nanorod for the NBE and DL peaks. (bottom) HAADF image of the nanorod. The green dotted line is added as an aid to the reader to show that the increase in the DL emission is coincident with an increase in HAADF signal. 